Research Paper

\title{
TMED2 promotes epithelial ovarian cancer growth
}

\author{
Gong Shi-Peng ${ }^{1, *}$, Chen Chun-Linn ${ }^{1, *}$, Wu Huan ${ }^{2,}{ }^{*}$, Meng Fan-Liang ${ }^{1}$, Chen Yong-Ning ${ }^{1}$, \\ Zhang Ya-Di ${ }^{1}$, Zhang Guang-Ping ${ }^{3}$ and Cai Ye-Ping ${ }^{4}$ \\ ${ }^{1}$ Department of Obstetrics and Gynecology, Nanfang Hospital, Southern Medical University, Guangzhou 510515, P.R. China \\ ${ }^{2}$ Department of Obstetrics and Gynecology, The Second Affiliated Hospital, Chongqing University of Medical Sciences, \\ Chongqing 400010, P.R. China \\ ${ }^{3}$ Department of Gynecology, People's Hospital of Huadu District, Guangzhou 510800, P.R. China \\ ${ }^{4}$ Department of Gynecology, Maternal and Child Health Hospital of Duanzhou District, Zhaoqing 526000, P.R. China \\ * These authors have contributed equally to the study and should considered as co-first authors \\ Correspondence to: Gong Shi-Peng, email: gsp2103@163.com \\ Keywords: epithelial ovarian cancer, TMED2, IGFIR, AKT, miR-30a
}

Received: August 06, $2017 \quad$ Accepted: September 08, $2017 \quad$ Published: October 06, 2017

Copyright: Shi-Peng et al. This is an open-access article distributed under the terms of the Creative Commons Attribution License 3.0 (CC BY 3.0), which permits unrestricted use, distribution, and reproduction in any medium, provided the original author and source are credited.

\section{ABSTRACT}

TMED2 is involved in morphogenesis of the mouse embryo and placenta. We found that expression of TMED2 was higher in epithelial ovarian cancer tissues than normal ovarian tissues. Silencing TMED2 decreased cell proliferation, migration, and invasion. Ectopic expression of TMED2 increased cell proliferation, migration and invasion. Silencing TMED2 inhibited ovarian cancer growth in mice. Silencing TMED2 inhibited IGF2/IGF1R/PI3K/Akt pathway. In agreement, ectopically expressed TMED2 activated IGF2/IGF1R/PI3K/Akt pathway. Mechanistic study revealed that TMED2 directly binds to AKT2, thereby facilitating its phosphorylation. We also found that TMED 2 increased IGF1R expression by competing for miR-30a. Thus, TMED2 is oncogenic and a potential target for epithelial ovarian cancer therapy.

\section{INTRODUCTION}

TMED2 is a member of the tranmembrane emp24 domain $[1,2]$. TMED2 was involved in the development of mouse embryos [3]. Previous study reported that ectopic expression of TMED2 can accelerate the proliferation in MC3T3-E1 cell [4]. This indicated its possible role in cancer cells proliferation. However, the role of TMED2 in epithelial ovarian carcinoma is still unknown. IGF1R is a transmembrane tyrosine kinase. It is often increased expression in many cancers. It promotes proliferation and inhibits apoptosis. IGF signaling pathway also mediates protease secretion, hypoxia signaling, cancer cell motility and adhesion. So, it is related to the invasion and metastasis in numerous cancers. Therefore, the IGF1R is a potential anti-cancer target. In some preclinical models of cancer, IGF1R antibody is showing promise $[5,6]$. Activate IGF2/PI3K/AKT signaling pathway promotes glioblastoma multiforme progression [7]. However, the role of TMED2 in IGF2/IGF1R/PI3K/AKT pathway is not elucidated yet.
In this research, we noted an elevated expression of TMED2 in epithelial ovarian carcinoma tissues. Our data also found that TMED2 promoted the ability of proliferation, migration, invasion in ovarian cancer cells. we also observed TMED2 can modulate IGF2/ IGF1R/ PI3K/AKT pathway. Further, we identified TMED2 directly binds to AKT2, thereby facilitating its phosphorylation. TMED2 served as a competing endogenous RNA (ceRNA) to regulate the expression of IGF1R through competing for miR-30a.

\section{RESULTS}

The expression of TMED2 was increased in epithelial ovarian cancer

We firstly analyzed the mRNA expression of TMED2 in ovarian carcinoma derived from Oncomine database. As showed in Figure $1 \mathrm{~A}(\mathrm{I})$, the mRNA expression of TMED2 in ovarian carcinoma was increased compared with normal ovarian tissues $(\mathrm{P}=8.91 \mathrm{E}-16)$. The 
expression of TMED2 was increased in ovarian serous cystadenocarcinoma compared with normal ovarian tissues(Figure 1A(II); $\mathrm{P}=5.21 \mathrm{E}-5)$. The expression of TMED2 was increased in ovarian mucinous adenocarcinoma, ovarian serous adenocarcinoma and ovarian endometrioid adenocarcinoma was elevated compared to normal ovarian tissues(Figure 1A(III-V); $\mathrm{P}=0.043,0.016$ and 0.049 respectively). However, the expression difference of TMED2 between ovarian clear cell adenocarcinoma and normal ovarian tissues is not significant $(\mathrm{P}=0.102)$. We next analyzed the expression and location of TMED2 in ovarian carcinoma tissues derived from Human Protein Atlas. As showed in Figure 1B, TMED2 was located in cytoplasmic and membranous.
The TMED2 in ovarian mucinous adenocarcinoma, ovarian serous adenocarcinoma and ovarian endometrioid adenocarcinoma was moderate expression(Figure 1B(IIII)). The TMED2 in follicle cells was medium expression. However, the TMED2 was not detected in ovarian stroma cells(Figure 1B(IV)).

We then determined the expression and location of TMED2 in epithelial ovarian carcinoma tissues and normal ovarian tissues. The expression of TMED2 was elevated in epithelial ovarian carcinoma tissues compared to that in normal ovarian tissues(Figure 1C). TMED2 was mainly localized on the cell membrane and cytoplasm (Figure 1C(I-III)). In this research, we found an increased expression of TMED2 in epithelial

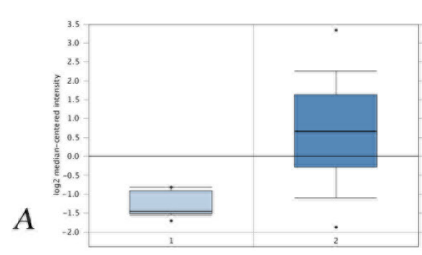

(I)

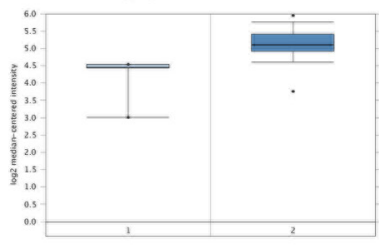

(IV)

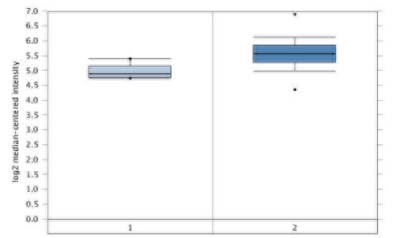

(II)

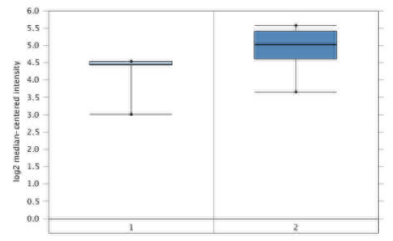

(

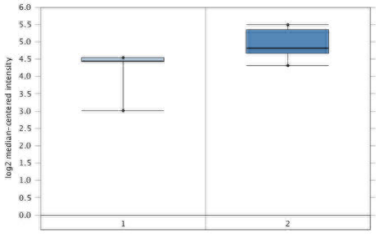

(III)

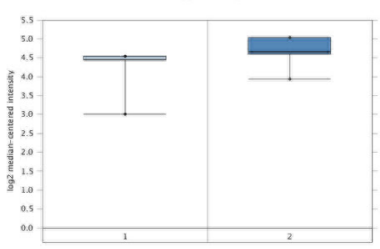

$(V I)$

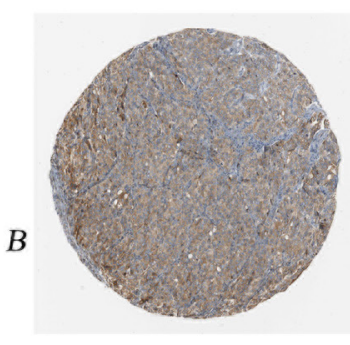

(I)

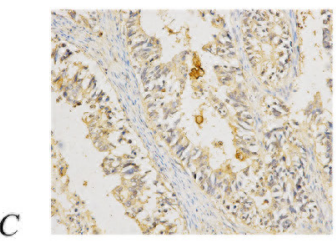

(I)

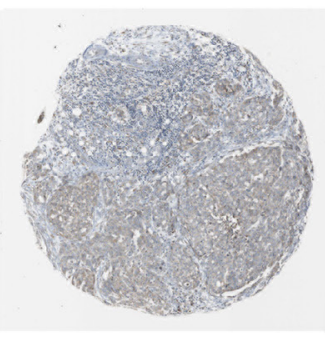

(II)

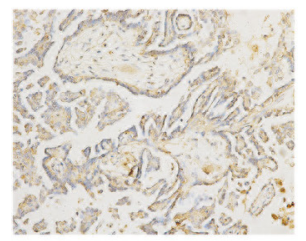

(II)

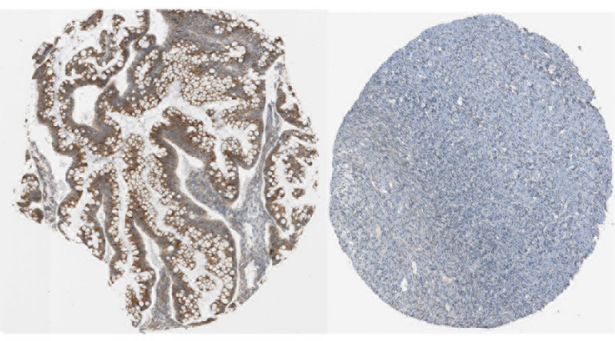

(III)

(IV)

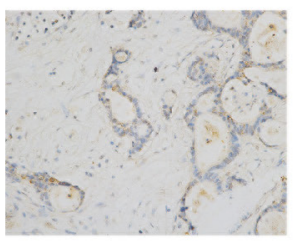

(III)

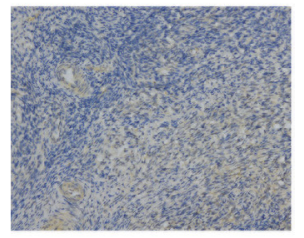

(IV)

Figure 1: Immunohistochemical analysis of TMED2 expression in ovarian cancer. The mRNA expression of TMED2 in ovarian cancer was analyzed. Data derived from Oncomine database. The mRNA expression of TMED2 in ovarian cancer was increased compared to normal ovarian tissues (A(I-II)). The expression of TMED2 was increased in ovarian mucinous adenocarcinoma, ovarian serous adenocarcinoma and ovarian endometrioid adenocarcinoma was elevated compared to normal ovarian tissues (AIII-V). TMED2 was located in cytoplasmic and membranous (B). The TMED2 in ovarian mucinous adenocarcinoma, ovarian serous adenocarcinoma and ovarian endometrioid adenocarcinoma was moderate expression(BI-III). The expression of TMED2 was determined in endometrioid adenocarcinoma (CI), serous papillary adenocarcinoma (CII), mucinous adenocarcinoma (CIII) and normal ovarian tissues (CIV). Original magnification, 200X. 
Table 1: Association of TMED2 expression with clinicopathological characteristics in 148 patients of EOC

\begin{tabular}{lccc}
\hline & No. of patients & TMED2 expression \\
\cline { 2 - 4 } & $(\mathbf{n = 1 4 8 )}$ & Low no.(\%) & High no.(\%) \\
\hline Characteristics & & & \\
Age(years) & & & \\
$<50$ & 78 & $30(38.46 \%)$ & $48(61.54 \%)$ \\
$\geq 50$ & 70 & $33(47.14 \%)$ & $37(52.86 \%)$ \\
Normal ovarian & 20 & $16(80.00 \%)$ & $4(20.00 \%)$ \\
Cancer tissues & 128 & $47(36.72)$ & $81(63.28 \%)$ \\
FIGO stage & & & $<0.05$ \\
I/II & 98 & $6162.24 \%)$ & $37(37.76 \%)$ \\
III/IV & 30 & $2(6.67 \%)$ & $28(93.33 \%)$ \\
Grade & & & $<0.05$ \\
1 & 28 & $23(82.14 \%)$ & $5(17.86 \%)$ \\
2 & 34 & $22(64.71 \%)$ & $12(35.29 \%)$ \\
3 & 66 & $24(36.36 \%)$ & $42(63.64 \%)$ \\
\hline
\end{tabular}

ovarian carcinoma tissues compared to that in normal ovary tissues $(\mathrm{P}<0.05$; Figure $1 \mathrm{C}$ and Table 1$)$. Then, we determined the relationship between TMED2 expression and clinicopathologic variables of 148 epithelial ovarian carcinoma tissues (Table 1). Significantly higher expression of TMED2 was observed in advanced stages than those in the early stages $(\mathrm{P}<0.05)$. Further, the expression of TMED2 was related to the cancer grade $(\mathrm{P}<0.05)$. However, there was no significant correlation between TMED2 expression and age $(\mathrm{P}>0.05$; Table 1$)$.

\section{TMED2 regulates cellular proliferation}

We firstly investigated the mRNA level of TMED2 in six ovarian cancer cell lines using qPCR. We found that TMED2 was up-regulated in SKOV3 and ES-2 cell lines. At the same time, TMED2 was down-regulated in A2780 cell line(Figure 2A). Thus, SKOV3 cells were used to silence TMED2 expression, A2780 cells were used for ectopic expression of TMED2. To study the function of TMED2 in SKOV3 cells, we silenced the expression of the TMED2 gene. The expression of TMED2 was decreased after infection of LV3-1 or LV3-2 in SKOV3 cells (Figure 2A and 2B). We found that down-regulation of TMED2 after infection of LV3-1 and LV3-2 suppressed cell proliferation in SKOV3 cells $(\mathrm{P}<0.05$; Figure $2 \mathrm{C}$, $2 \mathrm{D}$ and $2 \mathrm{E}$ ). We also found that the proliferation ability was higher in LV5-TMED2 infected A2780 cells than that in LV-5-GFP-infected cells $(\mathrm{P}<0.05$; Figure $2 \mathrm{~F}, 2 \mathrm{G}$ and $2 \mathrm{H})$.

\section{TMED2 regulated cellular migration, invasion in vitro}

In order to determined the role of TMED2 in cell metastasis and invasion, wound healing assay and matrigel invasion assay were performed. Our results showed that the migration and invasion ability of LV3-1 or LV3-2 infected SKOV3 cells were reduced compared to that of LV3-NC infected SKOV3 cells $(\mathrm{p}<0.05)$ (Figure 3A and $3 \mathrm{~B})$. However, the migration and invasion ability of LV5TMED2 infected A2780 cells were increased compared to that of LV5-GFP infected A2780 cells $(\mathrm{P}<0.05)$ (Figure $3 \mathrm{C}$ and $3 \mathrm{D})$.

Cytoskeleton plays a very important role in the invasion and metastasis of tumor cells [8]. Therefore, we determined whether TMED2 regulated the cytoskeleton rearrangement using phalloidin staining. We found that F-actin was mostly localized in the cellular projections and outgrowth. Silencing TMED2 expression in SKOV3 cells, the formation of lamellipodia and membrane ruffles was suppressed(Figure 3E). A2780 cells infection of LV5-GFP showed some small ruffles. However, ectopic expression of TMED2 in A2780 cells induced F-actin reorganization at lamellipodia and membrane ruffles(Figure $3 \mathrm{~F}$ ).

\section{TMED2 regulation of IGF1R is $\mathrm{miR-30a}$ dependent}

Bioinformatics analyses (Targetscan) showed that IGF1R is a putative ceRNA of TMED2. We observed that 

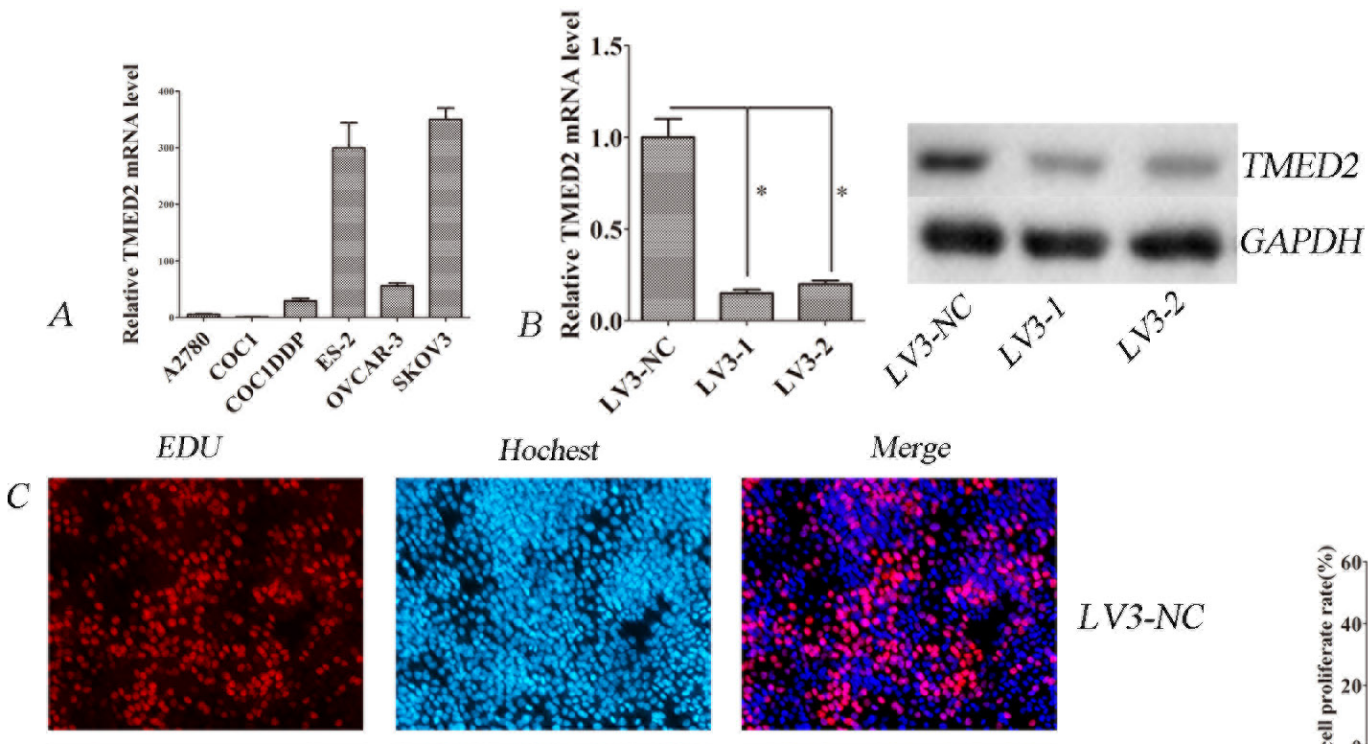

LV3-NC
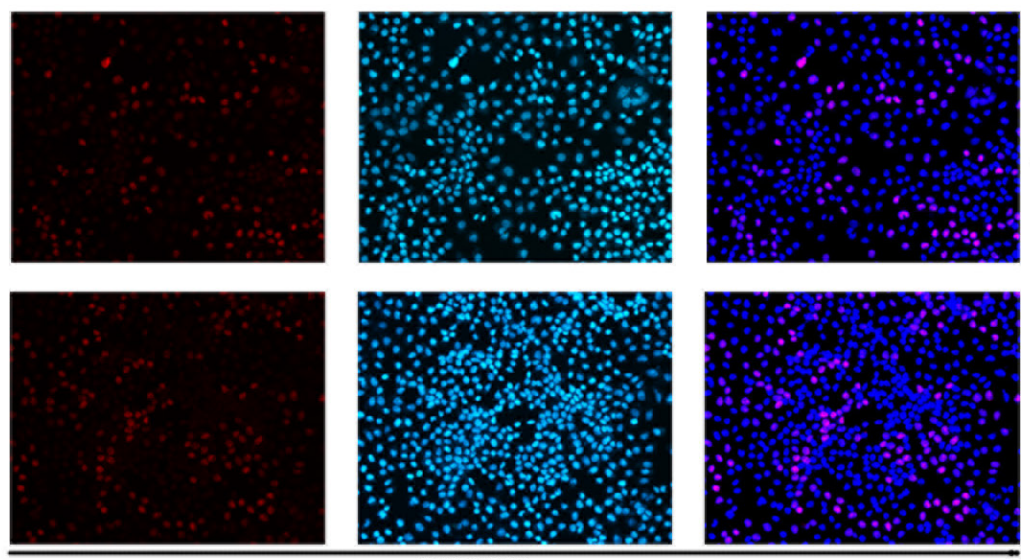

LV3-2

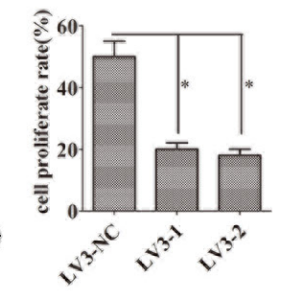

LV3-1
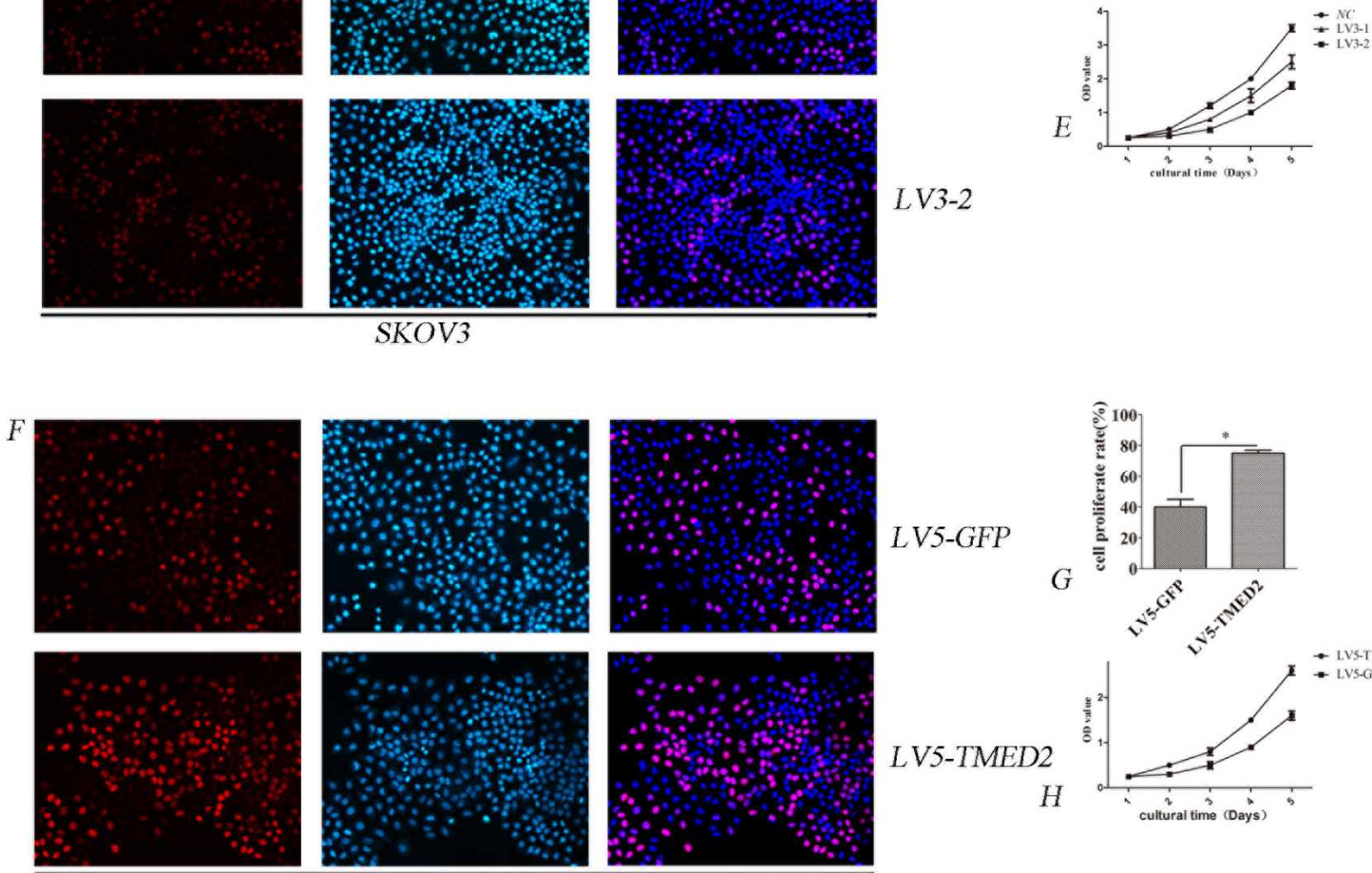

A2780

Figure 2: TMED2 regulates cellular proliferation. (A) The relative expression of TMED2 mRNA in ovarian cancer cell lines. (B) TMED2 mRNA and protein level were down-regulated by infected with LV3-1 or LV3-2. (C, D) Ovarian cancer SKOV3 cells were infected with LV3-NC, LV3-1 and LV3-2. Cell proliferation was assessed by EdU. The proliferation rate of LV3-1 and LV3-2 cells was lower than that of LV3-NC cells. Original magnification, 200X. (E) Cell proliferation was determined by CCK-8 assay. (F, G) Ovarian cancer A2780 cells were infected with LV5-GFP or LV5-TMED2. Then cell proliferation was determined by EdU. Original magnification, 200X. The proliferation rate of LV5-TMED2 cells was increased compared with LV5-GFP cells. (H) Cell proliferation was determined by CCK-8 assay. Error bars represent standard error. ${ }^{*} \mathrm{p}<0.05$, and ${ }^{* *} \mathrm{p}<0.001$. 

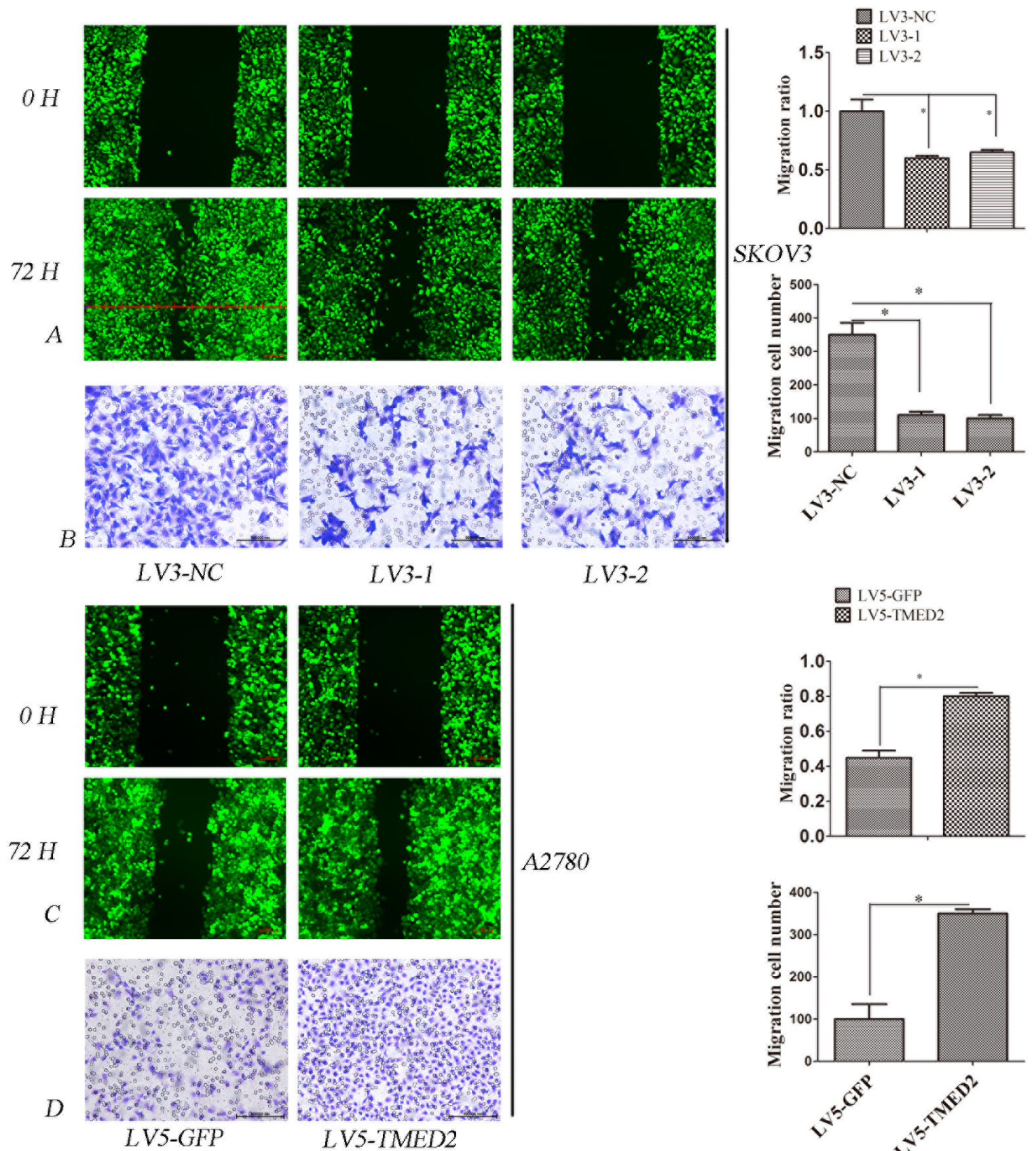

A2780
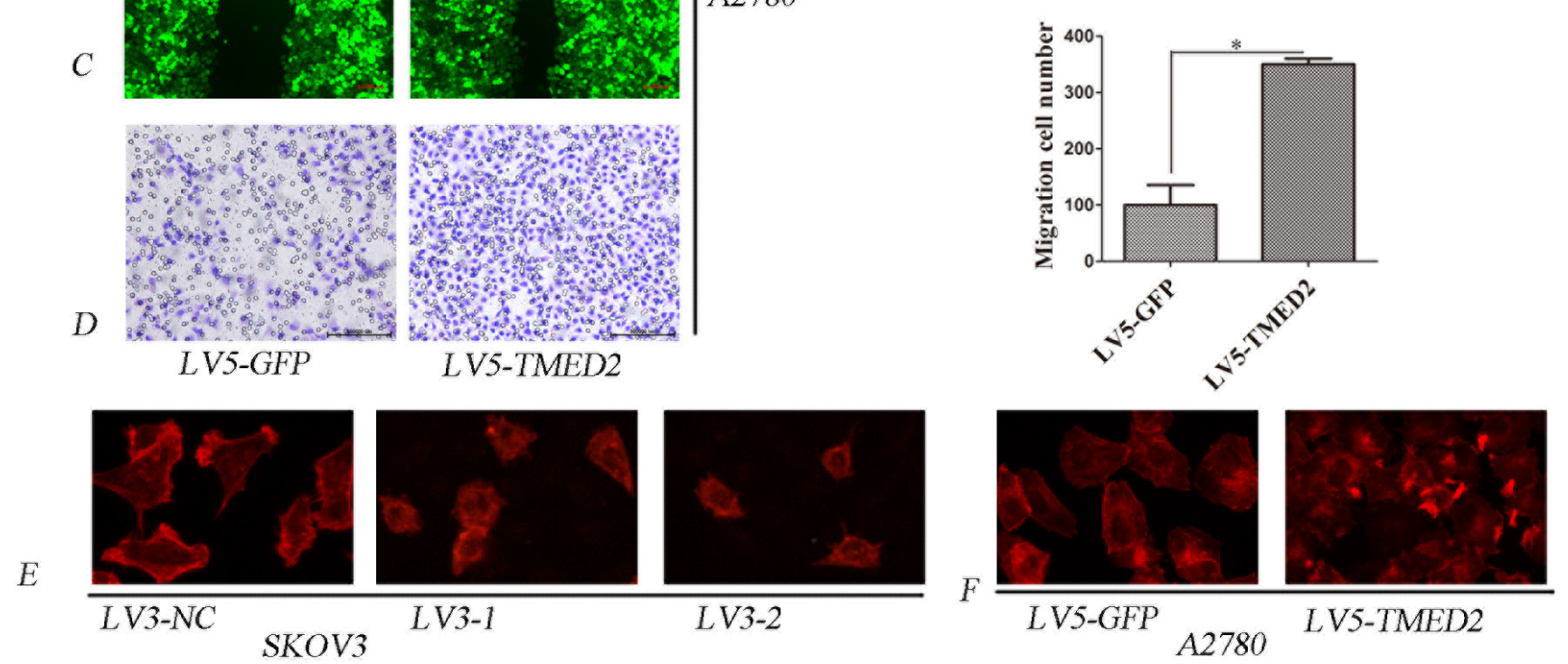

Figure 3: (A) Ovarian cancer SKOV3 cells migration ability were detected by the wound healing assay. The migration of LV3-1 and LV3-2 infected SKOV3 cells was lower as compared with LV3-NC infected cells. (B) Ovarian cancer SKOV3 cells invasion ability was detected by Matrigel invasion assays. The invasion ability of LV3-1 and LV3-2 infected SKOV3 cells was decreased compared with LV3NC infected cells. (C) Ovarian cancer A2780 cells migration ability was detected by wound healing assay. The migration ability of LV5TMED2 infected A2780 cells was increased compared with LV3-NC infected cells. (D) Ovarian cancer A2780 cells invasion ability was detected by Matrigel invasion assays. The invasion ability of LV5-TMED2 infected A2780 cells was increased compared with LV5-GFP infected cells. (E and F) F-actin staining. Original magnification, 400X. Error bars represent standard error. ${ }^{*} \mathrm{p}<0.05$, and ${ }^{* *} \mathrm{p}<0.001$. 

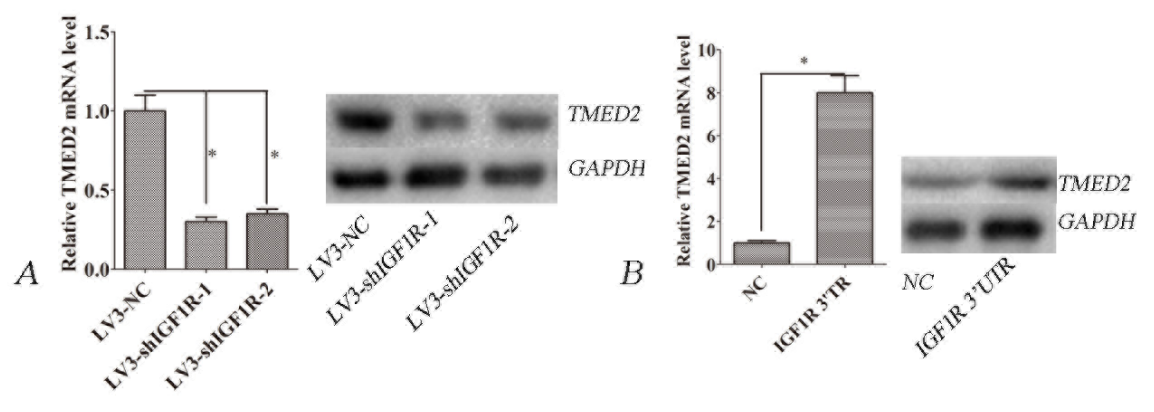

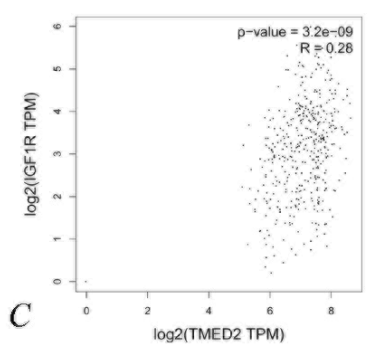

IGF1R 3'UTR 5'-GUUGACGAAGAUCUGGUUUACAA-3'

(Position 5629-5635)

TMED2 3' UTR 5'-CAACUCAGAAUUCACUGUUUACC-3'

(Position 37-43)

hsa-miR-30a-5p 3'-GAAGGUCAGCUCCUACAAAUGU-5',
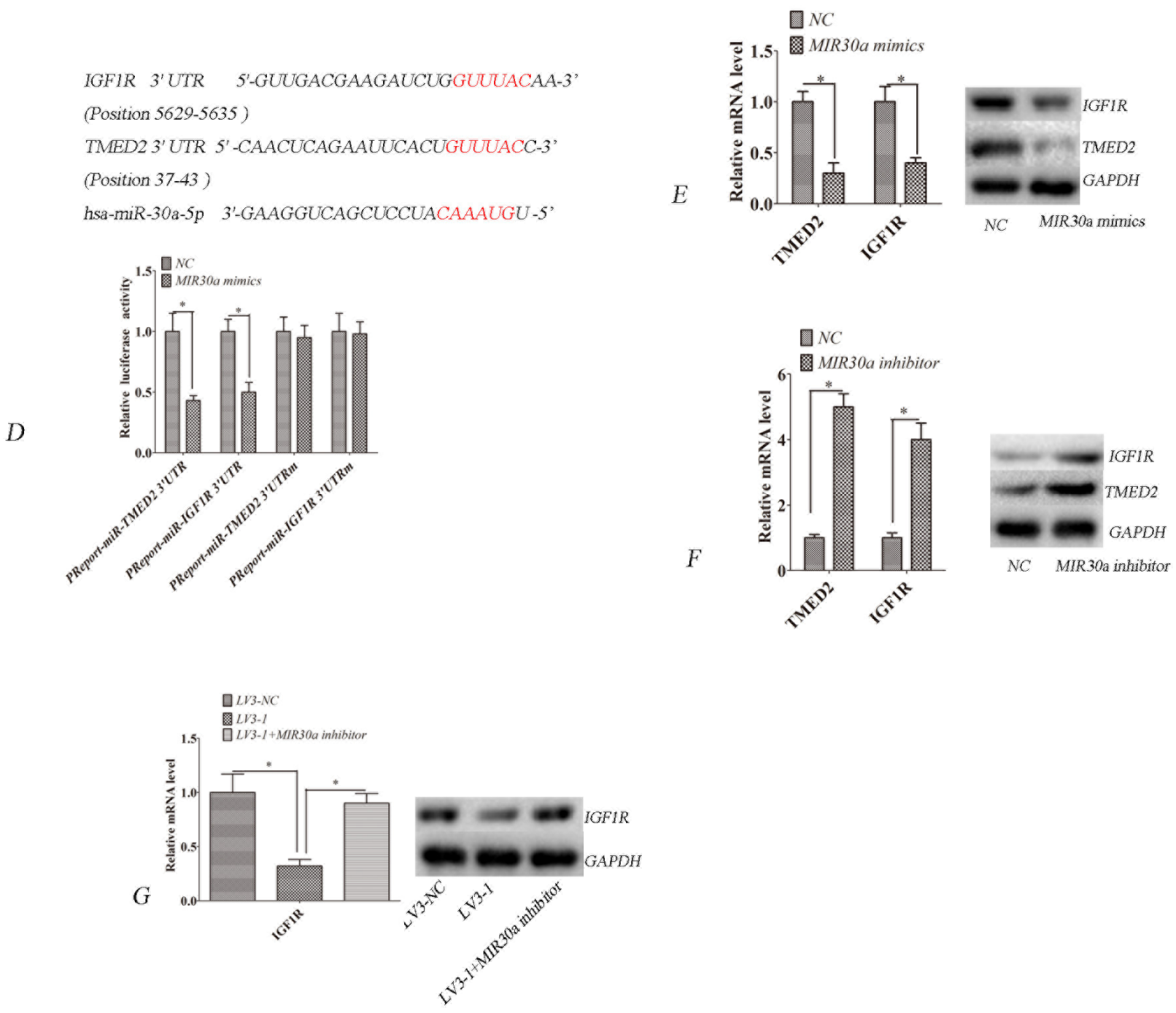

Figure 4: TMED2 regulates IGF1R mRNA and protein levels. (A) TMED2 mRNA and protein levels were downregulated by infection with LV3-shIGF1R-1 and LV3- shIGF1R-2. (B) Ovarian cancer SKOV3 cells were transfected with IGF1R 3'UTR and negative control. TMED2 mRNA and protein levels were increased by transfection with IGF1R 3'UTR. (C) The expression of TMED2 in ovarian cancer was positively correlated with IGF1R. Data derived from the The TCGA database (R=0.28; P=3.2E-09; http://gepia.cancer-pku. cn/detail.php). (D) Putative binding sites targeted by miR-30a were predicted to be located in the 3' UTR of TMED2 and IGF1R mRNA. SKOV3 cells were cotransfected with miR-30a mimics or control RNA (NC) with luciferase reporter plasmids containing either wildtype (pMIR-TMED2-3UTR and pMIR-IGF1R-3UTR) or mutant 3' UTR (pMIR-TMED2-3UTRm and pMIR-IGF1R-3UTRm) of TMED2 and IGF1R genes. Luciferase expression was measured. The fold changes of the relative luciferase activity in miR-30a mimics with the indicated plasmids transfected cells were normalized to $\mathrm{NC}$ with the corresponding indicated plasmid-transfected cells. (E) Ovarian cancer SKOV3 cells were transfected with miR-30a mimics or control RNA (NC). (F) Ovarian cancer SKOV3 cells were transfected with miR30a inhibitor or control RNA (NC). (G) Ovarian cancer SKOV3 cells were infected with LV3-NC and LV3-1. One group was infected with LV3-1-transfected miR-30a inhibitors. Error bars represent standard error. The symbols ${ }^{*}$ and ${ }^{* *}$ indicate $p<0.05$ and 0.01 , respectively. 
IGF1R mRNA and protein expression were obviously reduced in LV3-shIGF1R-1 or LV3-shIGF1R-2 infected SKOV3 cells compared to LV3-NC infected SKOV3 cells (Figure 4A; $\mathrm{P}<0.05$ ). However, the mRNA and protein expression of IGF1R were upregulated when the TMED2 3' untranslated region (UTR) was ectopically expressed in A2780 cells (Figure 4B; $\mathrm{P}<0.05$ ). The TCGA data indicated that the expression of TMED2 in ovarian cancer was positively correlated with IGF1R (Figure 4C, $\mathrm{R}=0.28 ; \mathrm{P}=3.2 \mathrm{E}-09$ )(http://gepia.cancer-pku. cn/detail.php)[9].

We investigated whether the effect of TMED2 on IGF1R is miRNA dependent. Interestingly, bioinformatics analyses showed that TMED2 and IGF1R are predictive targets of miR-30a (Targetscan). Thus, we attempted to experimentally verify whether miR-30a modulated TMED2 and IGF1R expressions in SKOV3 cells. Bioinformatics predicted that there was a miR-30a binding site in the 3' UTR of TMED2 and IGF1R mRNAs (Figure 4D). We constructed a vector to determine whether miR30 a can directly target the 3' UTR of TMED2 and IGF1R. Our results showed that miR-30a mimics significantly suppressed luciferase activity of the reporter vector. In contrast, no significant suppression of luciferase activity was detected in cells transfected with the control vector with mutant TMED2 and IGF1R 3' UTR when miR$30 \mathrm{a}$ expression was elevated (Figure 4D; $\mathrm{P}<0.05$ ). miR$30 \mathrm{a}$ mimics could reduce the expression of TMED2 and IGF1R (Figure 4E; $\mathrm{P}<0.05$ ), whereas miR-30a inhibitor could elevate the expression of these genes (Figure 4F; $\mathrm{P}<0.05)$. These data indicate that TMED2 and IGF1R are direct targets of miR-30a. Furthermore, we found that TMED2's regulation of IGF1R was eliminated when miR-30a was inhibited (Figure 4G; $\mathrm{P}<0.05$ ). These data indicate that TMED2's regulation of IGF1R is miR-30a dependent.

\section{TMED2 regulated IGF1R/IGF2/PI3K/AKT pathway}

Bioinformatics analyses (https://thebiogrid.org/) showed that TMED2 may interacted with AKT2(Figure $5 \mathrm{~A})$. In order to identify the interaction between TMED2 and AKT2, we designed a Co-immunoprecipitation (CoIP) experiment. Cells were co-transfected with FlagAKT2 and HA- TMED2. Control group was established simultaneously, and then were harvested $24 \mathrm{~h}$ later. AntiHA antibodies were used to pull the interaction protein. Then, they were detected by anti-Flag antibodies using Western blot. Our results indicated that Flag bands could
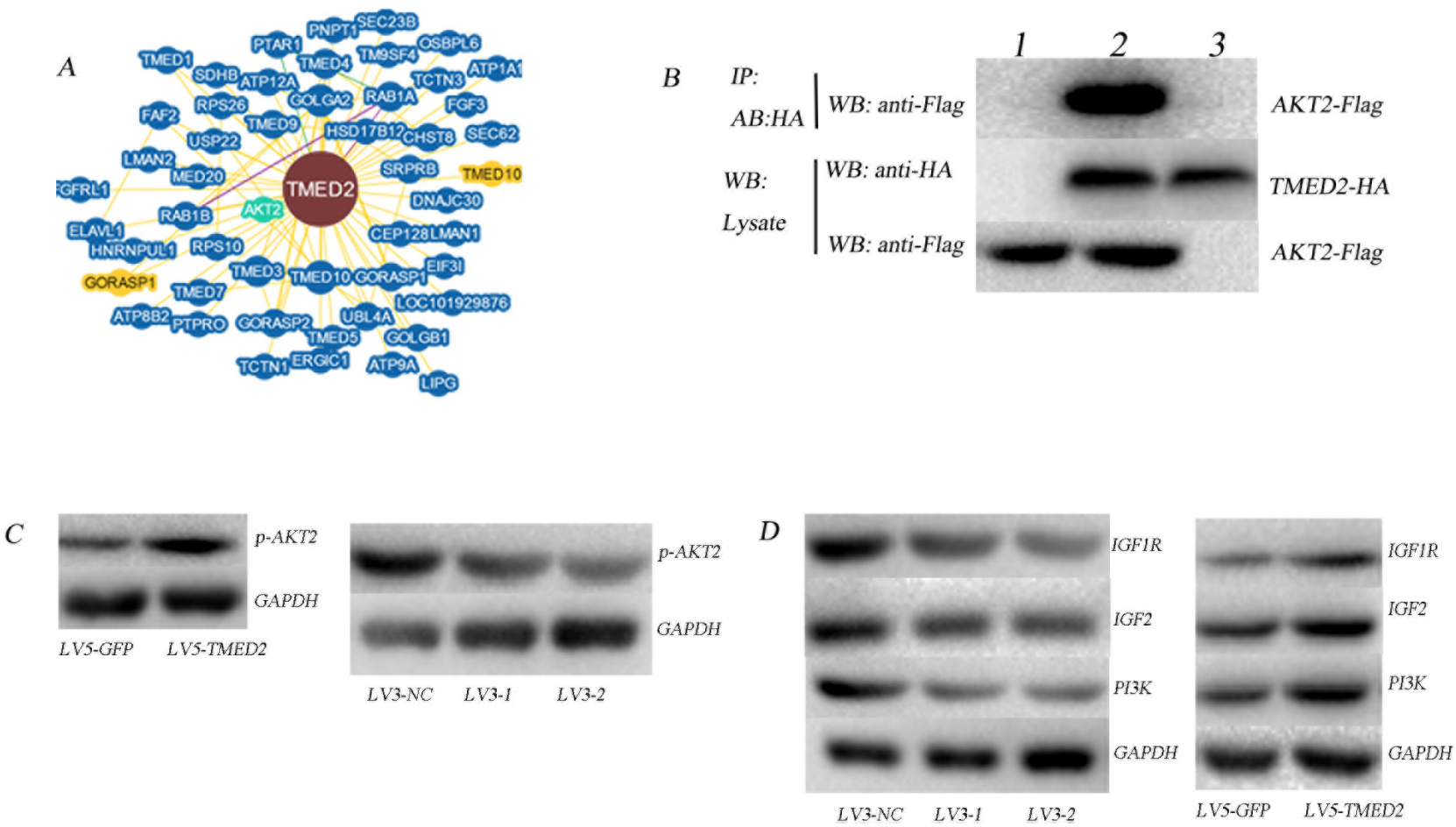

Figure 5: (A) Bioinformatics analyses showed that TMED2 may interacted with AKT2 (https://thebiogrid.org/). (B) The interaction between TMED2 and AKT2 was identified by Co-IP assay. Cells were co-transfected with Flag-AKT2 and HA-TMED2, and control group was established simultaneously, cells were then harvested $24 \mathrm{~h}$ later. Anti-HA antibodies were used to pull the interaction protein. Then, they were detected by anti-Flag antibodies. Results showed that Flag bands could not be detected in the cells transfected with Flag-AKT2 (lane 3) or HA- TMED2 (lane 1) only. However, it can be detected in cells co-transfected with both Flag-AKT2 and HA-TMED2 (lane 2), which indicated that there existed interaction between TMED2 and AKT2 in vivo. (C-D) The expression of P-AKT2, IGF1R, IGF2 and PI3K was determined by Western Blotting. 
not be detected in cells infected with Flag-AKT2 or HATMED2 only. However, It can be detected in cells that co-transfected with Flag-AKT2 and HA-TMED2(Figure $5 \mathrm{~B})$. We also observed that overexpression of TMED2 increased the expression of p-AKT2; however, silencing TMED2 decreased the expression of p-AKT2(Figure 5C). This data suggested that TMED2 directly binds to AKT and promoted its phosphorylation.

Previous studies suggested that IGF2 participate in the malignant behavior of ovarian cancer through PI3K/ Akt signal pathway $[10,11]$. Based on the above results, we guessed TMED2 may regulated the IGF1R/IGF2/PI3K/ AKT pathway. The expression of IGF1R, IGF2, PI3K and p-AKT was reduced in SKOV3 cells infected with LV3-
1 or LV3-2. In contrast, the expression of IGF1R, IGF2, PI3K and p-AKT was increased in A2780 cells infected with LV5-TMED2 (Figure 5D).

TMED2 promoted A2780 cellular proliferation, migration and invasion involves in IGF1R/IGF2/ PI3K/AKT pathway

We have demonstrated that TMED2 can regulate the IGF1R/IGF2/PI3K/AKT signaling axis. Hence, we presumed that TMED2 regulation of epithelial ovarian cancer proliferation, migration and invasion involves IGF1R/IGF2/PI3K/AKT pathway. Our data showed that the ability of cellular proliferation, migration and

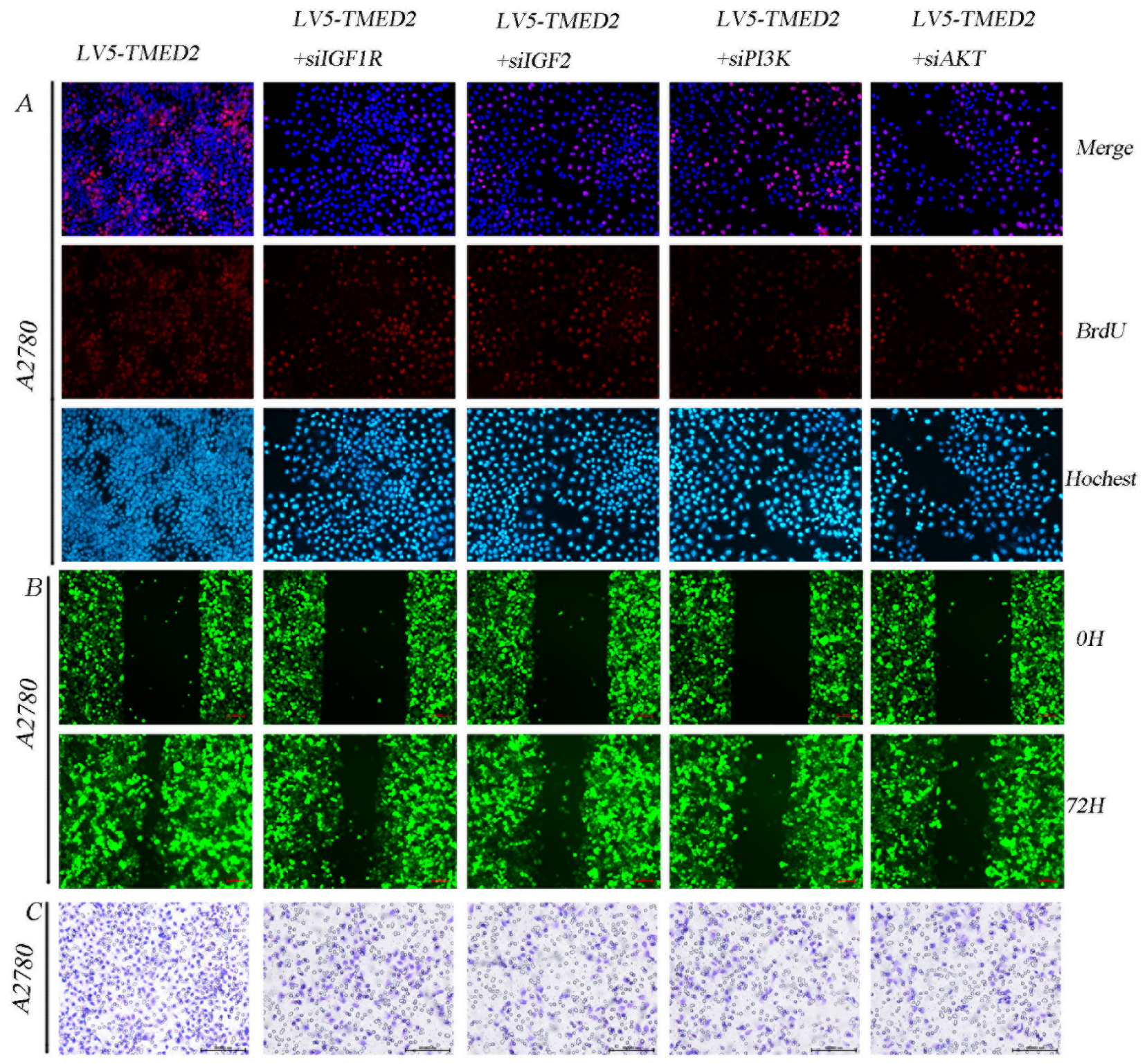

Figure 6: TMED2 promoted A2780 cellular proliferation, migration and invasion involves IGF1R/IGF2/PI3K/AKT pathway. A2780 cells infected with LV5-GFP and LV5-TMED2. After $48 \mathrm{~h}$, puromycin was added at a concentration of $2.5 \mu \mathrm{g} / \mathrm{ml}$. The cells were transfected with IGF1R siRNA, IGF2 siRNA, PI3K siRNA or AKT siRNA for $48 \mathrm{~h}$. Then the cellular proliferation ability (A), migration ability (B) and invasion ability (C) were detected. Original magnification, 200X. 


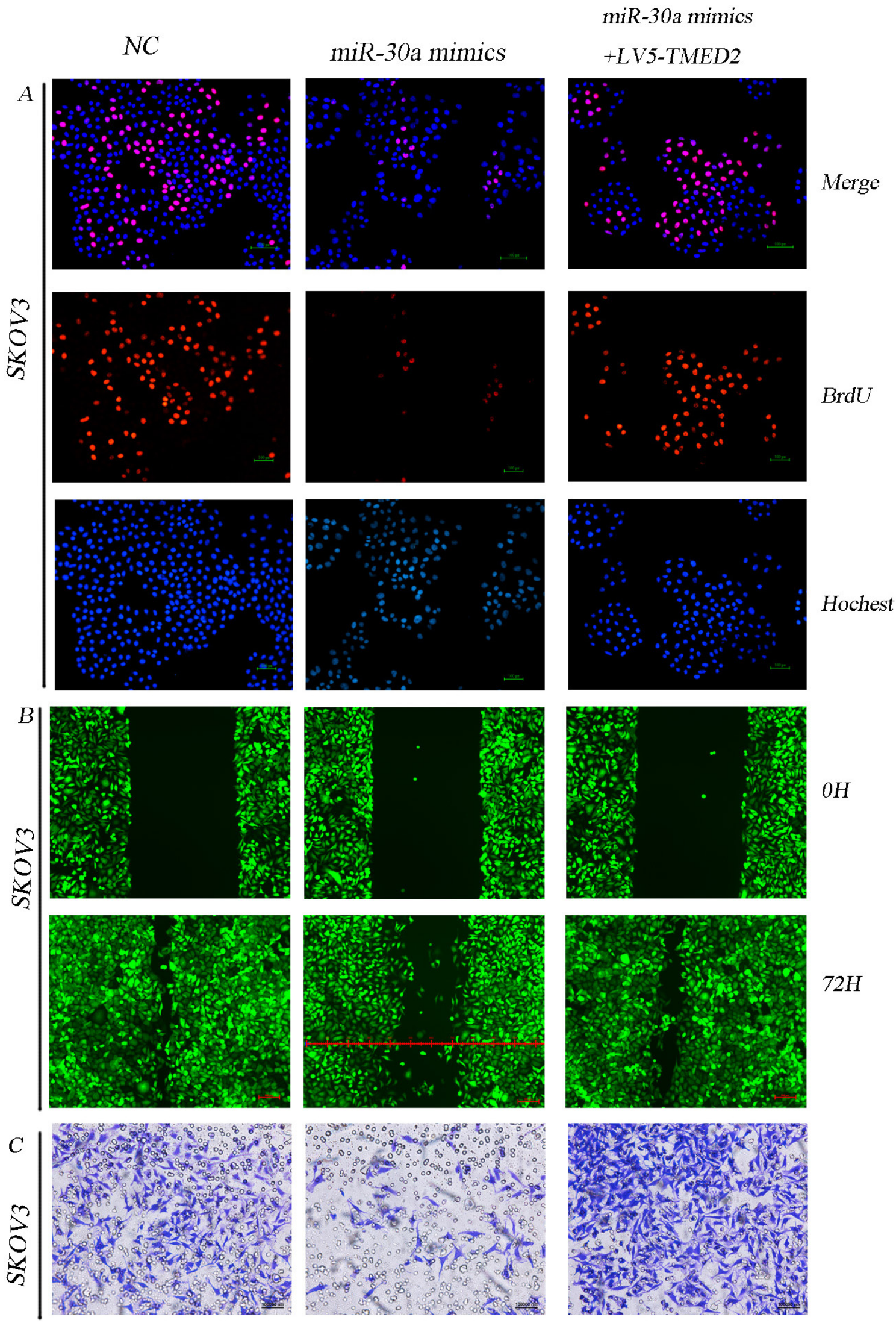

Figure 7: miR-30a regulated SKOV3 cellular proliferation, migration and invasion through directly targeting TMED2. Ovarian cancer SKOV3 cells were transfected with NC(negative control), miR-30a mimics or LV5-TMED2 + miR-30a mimics. Then the cellular proliferation ability (A), migration ability (B) and invasion ability (C) were detected. Original magnification, 200X. 
invasion was elevated in A2780 cells infected with LV5TMED2 compared to A2780 cells infected with LV5GFP. Silencing of IGF1R, IGF2, PI3K or AKT partially abrogated this effect of TMED2 overexpression (Figure 6A-6C).

\section{miR-30a regulated $S K O V 3$ cellular proliferation, migration and invasion through directly targeting TMED2}

We have demonstrated TMED2 is a directly target. So, we investigated whether miR-30a regulated the malignant behavior through targeting TMED2 in SKOV3 cells. miR-30a mimics inhibited the malignant behavior in ovarian carcinoma SKOV3 cells. Ectopic expression of TMED2 can partially restored those phenotypes (Figure 7A-7C).

\section{Silencing of TMED2 retarded the growth of ovarian cancer SKOV3 cells in vivo}

The regulation of TMED2 in the growth of ovarian cancer SKOV3 cells in vivo was determined using tumor xenografts model. The average volume of tumors was reduced in SKOV3 cells infected with LV3-1 compared to that of SKOV3 cells infected with LV3-NC $(\mathrm{P}<0.05)$

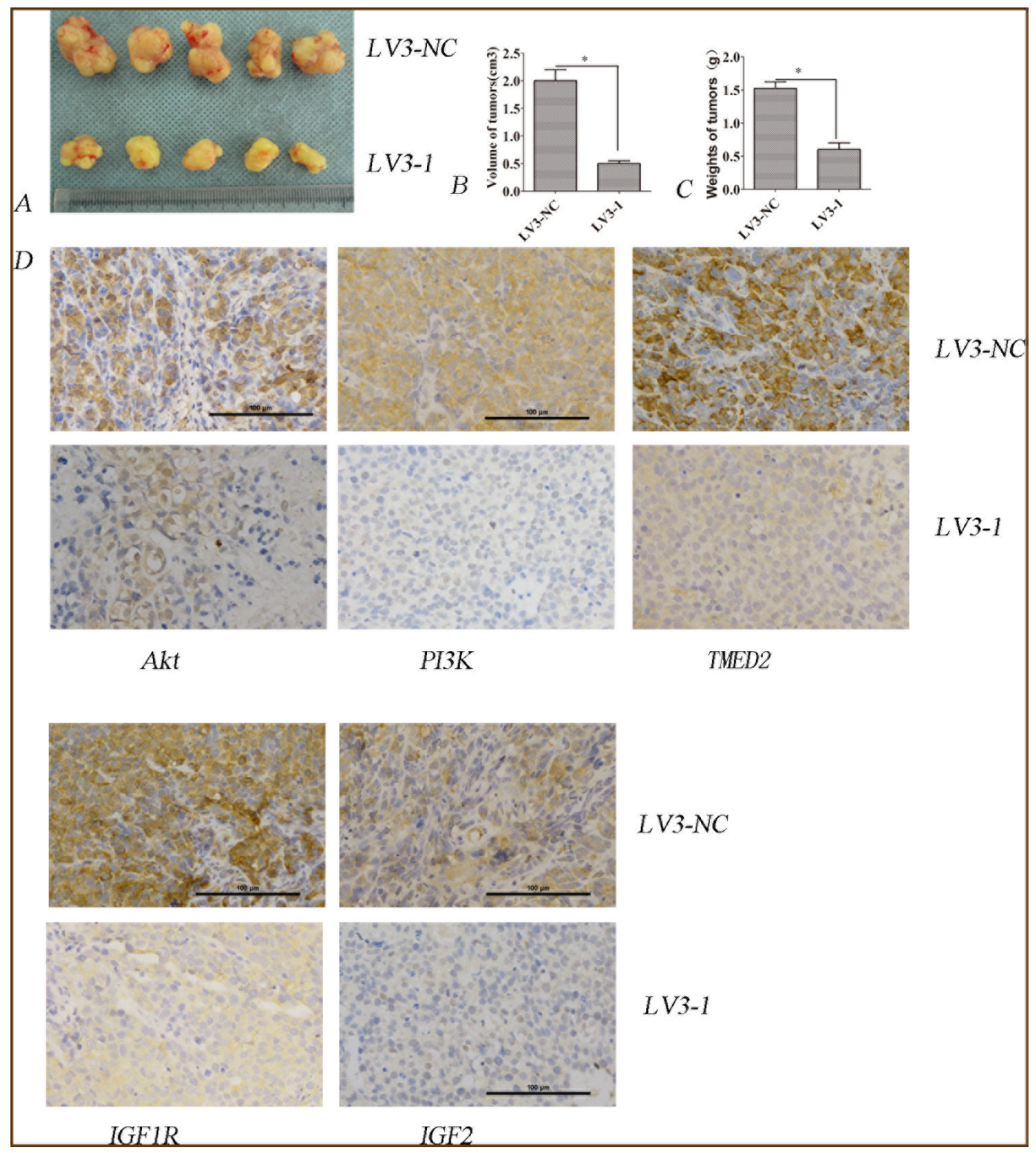

Figure 8: TMED2 regulated tumorigenesis in nude mice model. (A, B and C) Mean tumor volume and weight on day 28 after tumor cell injection. LV3-1 or LV3-NC infected SKOV3 cells were implanted s.c. into the left armpit. (D). Immunohistochemical analysis of TMED2, IGF1R, IGF2, PI3K or p-AKT expression were performed on tumor xenografts. Representative images are shown (original magnification $\times 200$ ). ${ }^{*} \mathrm{p}<0.05$, and ${ }^{* *} \mathrm{p}<0.001$. 
(Figure $8 \mathrm{~A}$ and $8 \mathrm{~B}$ ). The average weight of tumors in SKOV3 cells infected with LV3-1 was reduced compared to that of SKOV3 cells infected with LV3-NC $(\mathrm{P}<0.05$; Figure 8C). The expression of TMED2, IGF1R, IGF2, $\mathrm{PI} 3 \mathrm{~K}$ or $\mathrm{p}-\mathrm{AKT}$ in tumors from SKOV3 cells was reduced when silencing TMED2 ( $\mathrm{P}<0.05$; Figure $8 \mathrm{D})$.

\section{DISCUSSION}

In our research, we observed an increased expression of TMED2 in epithelial ovarian carcinoma compared with the normal ovarian tissues. The high expression of TMED2 in epithelial ovarian cancer was related to the histological grades and cancer stage. We also found that the elevated expression of TMED2 in epithelial ovarian carcinoma promoted proliferation and invasion. Our results also demonstrate that TMED2 can regulate IGF1R in a miR-30a-dependent manner (Figure 9). Based on these findings, TMED2 can serve as a new ovarian cancer biomarkers and a potential cancer treatment target.

Competing endogenous RNAs (ceRNAs) is a hypothesis that transcripts regulate each other by competing for the same miRNAs [12]. CeRNA-like regulation, including regulators, targets, modulators that can be non-coding RNAs, proteins, and even DNA regions, can regulate gene expression [13]. The ceRNA effect consists of a positive effective interaction that can arise between transcripts that are targeted by the same miRNA species due to competition [14]. Many studies suggested that ceRNAs were involved in a lot of biological processes, such as post-transcriptional regulation of gene expression [15]. To explore the functions of TMED2 in epithelial ovarian cancer, we predicted the candidate ceRNAs of TMED2 by bioinformatics analyses [16]. Our results indicate that TMED2 and IGF1R regulate each other through competition for miR-30a. We also found that the expression of TMED2 was correlated with IGF1R.

IGF1R is a transmembrane tyrosine kinase which is usually increased expression in several caner types. It promotes cellular proliferation and inhibits apoptosis in cancer cells. IGF participates in many signal pathway including PI3K-AKT pathway, cAMP-PKA pathway and MEK-ERK1/2 pathway. Therefore, the IGF1R is a potential cancer treatment target $[5,6]$. Our data showed that TMED2 is a ceRNA of IGF1R. We also demonstrated that silencing IGF1R can partly inhibited the malignant behavior of A2780 cells induced through ectopic expression of TMED2. So we assumed that inhibited the IGF1R pathway may be a potential target of patients who over-expression of TMED2.

$\mathrm{AKT}$ is at the hub of some signal pathway. It promotes the malignant behavior of cancer [17]. A previous study

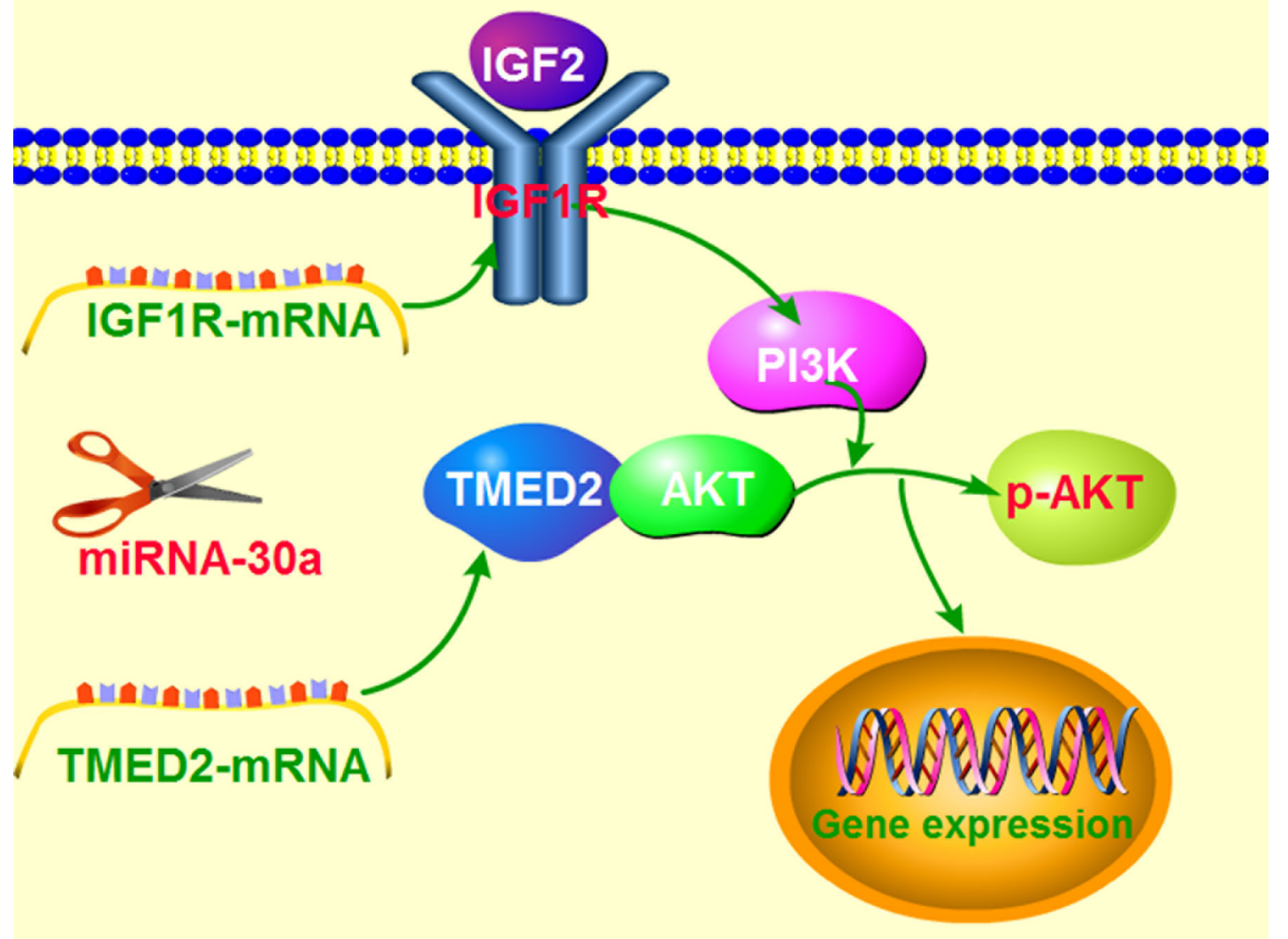

Figure 9: Schematic model demonstrating TMED2 function in ovarian cancer and potential mechanisms. 
demonstrated that AKT was up-regulated in ovarian cancer tissues and cell lines [18]. Inhibition of AKT activation sensitized cell survival in cisplatin resistant epithelial ovarian cancer $[19,20]$. Our data indicated that TMED2 directly binds to AKT2, thereby facilitating its phosphorylation. So the over-expression of TMED2 maybe promote malignant behavior of ovarian cancer by activating AKT pathway. Activating IGF2/PI3K/AKT pathway promotes glioblastomas multiforme progression [7]. Our study found that TMED2 regulates epithelial ovarian cancer progression by activating IGF2/IGF1R/PI3K/AKT pathway. So we guess that inhibited this pathway maybe a potential therapy target in ovarian cancer which elevated expression of TMED2.

MiR-30a suppresses tumor metastasis and proliferate in many cancers [21-23]. miR-30a can regulate malignant behavior in ovarian cancer [24]. miR-30a regulated $\mathrm{PI} 3 \mathrm{~K} / \mathrm{AKT}$ pathway by directly target IGF1R in non-small cell lung cancer [25]. Our study also found that IGF1R is a directly target of miR-30a. So our finding is consistent with this report. We also determined that miR30 a regulated proliferate, migration and invasion through targeting TMED2. This data indicated that TMED2 was an tumor-promoting gene.

To sum up, this research found that TMED2 is a putative oncogene in ovarian cancer. It is related to IGF2/ IGF1R/PI3K/AKT pathway. This implicated that TMED2 is a potential treatment target in epithelial ovarian carcinoma.

\section{MATERIALS AND METHODS}

\section{Tissue specimens}

The tissue microarray slides containing malignant and benign ovarian tissues $(n=148)$ were obtained from US Biomax Inc cancer tissue bank collection (US Biomax Inc., MD, USA).

\section{Cell culture, transfection procedure, and reagents}

The A2780 cell line was established from tumor tissue from an untreated patient. The ES-2 cell line was established from a surgical tumor specimen taken from a 47 year old black woman. SKOV3 human ovarian cancer cell line derived from the ascites from a 64 year old caucasian female with an ovarian tumor. The COC1 cell line was derived from the ascites of patients with poorly differentiated ovarian cancer. Cisplatin-resistant COC1/ DDP, which is derived from its parental ovarian cancer cell line $\mathrm{COC} 1$ by stepwise selection in vitro using cisplatin. CAOV-3 human ovarian cancer cell line derived from a 54 years old caucasian female with ovarian adenocarcinoma. Human ovarian cancer cells were cultured in Rosewall Park Memorial Institute (RPM1)1640 medium containing $10 \%$ fetal bovine serum and antibiotics, and incubated in an atmosphere with $5 \%$ carbon dioxide at $37^{\circ} \mathrm{C}$. Doublestrand oligonucleotides corresponding to the target sequences were synthesized by Genepharma Co., Ltd. (Shanghai, China). The following sequences were targeted for human TMED2, IGF1R, IGF2, PI3K and AKT2 small interfering RNA (siRNA), respectively. TMED21:5'-AGCUAGAAGAAAUGAUCAAUG-3'; TMED22:5'- GGUUCAAGUCACUAAAGAUUC-3'; IGF1R: 5'-GC GGAGAGAUGUCAUGCAAGU-3'; IGF2: 5'-CGUGUU UGACUCAACUCAACU-3'; IGF2: 5'-GCGGAGAG AUGUCAUGCAAGU-3'; PI3K: 5'-GGAUCAAGUU GUCAAAGAAGA-3'; AKT2: 5'-GGUUCUUCCUCA GCAUCAACU-3' and NC (negative control) siRNA: 5'-UUCUUCGAAGGUGUCACGUTT-3'. Lentiviral vector expressing shRNA targeting TMED2 (named LV3-1 and LV3-2) and TMED2-lentiviral expression vector (named LV5-TMED2) were provided from Genepharma Co., Ltd. (Shanghai, China). miR-30a mimics were synthesized at Ruibo Biotech (Ruibo Biotechnology, Guangzhou, China).

\section{Immunohistochemistry}

Immunohistochemistry (IHC) was performed according to the SP kit instructions (SP-9000, ZSGB$\mathrm{BIO}$, Beijing, China). After dewaxing and hydration, the sections were heated in citrate buffer ( $\mathrm{pH}$ 6.0, SigmaAldrich, USA) in a microwave oven for 20 minutes for antigen retrieval. Further, the sections were cooled naturally to room temperature. The sections were washed thrice for three minutes per cycle. Subsequently, the sections were incubated in $3 \%$ aquae hydrogenii dioxidi for 15 minutes at room temperature, washed thrice with phosphate buffered saline (PBS) for 3 minutes per cycles. The sections were blocked with $5 \%$ goat serum (ab7481; Abcam Company) for 30 minutes at $37^{\circ} \mathrm{C}$. AntiTMED2 rabbit polyclonal antibody (1:100, Sigma) was incubated with the sections overnight at $4^{\circ} \mathrm{C}$. Negative controls included omission of primary antibody and use of irrelevant primary antibodies. The corresponding secondary antibodies that were conjugated to horseradish peroxidase (Bioss Biotechnology), were incubated with the sections for an hour at room temperature. The sections were washed thrice in PBS for 3 minutes per cycle. The sections were incubated in horseradish enzyme-labeled chain avidin solution (Bioss Biotechnology) for 30 minutes at $37^{\circ} \mathrm{C}$, and washed in PBS for 3 minutes $x 3$ cycles. The proteins were visualized by diaminobenzidine. All the sections were observed by three independent pathologists using a light microscope. The staining data were obtained from manually recorded reports. Staining intensity was graded on a 0-3 scale as follows: 0 (absence of staining), 1 (weakly stained), 2 (moderately stained), and 3 (strongly stained). The percentage of positive tumor cells was scored as follows: 0 (absence of tumor cells), $1(<33 \%$ tumor cells), 2 (33-66\% tumor cells) and 3 ( $>66 \%$ tumor cells). The staining score was calculated as the product of staining intensity score and the percentage score; this score ranged from 0 to 9 (absence, $\mathrm{IHC}=0$; weak, $0<\mathrm{IHC} \leq 4$; strong, $5 \leq \mathrm{IHC} \leq 9$ ) [26] 


\section{F-actin staining}

Ovarian cancer SKOV3 and A2780 cells were seeded $\left(1 \times 10^{4}\right)$ on the cover slip and allowed to adhere overnight. Twenty-four hours after transfection, cells were fixed, permeabilized and stained with Tetramethylrhodamine (TRITC)-conjugated phalloidin (Sigma-Aldrich, Louis, USA) for 2 hour. Nuclei were stained with Hochest (Sigma-Aldrich, Louis, USA) for $15 \mathrm{~min}$. The results were analyzed with fluorescence microscope.

\section{Quantitative real-time polymerase chain reaction(PCR)}

Total RNA was isolated using a RNA pure Highpurity Total RNA Rapid Extraction Kit (BioTeke, RP1201, China), as per the instructions provided in the kit. cDNA was synthesized using the iSCRIPT cDNA synthesis kit (Bio-Rad). The primers used for amplifying TMED2, IGF1R and GAPDH were synthesized by Guangzhou Funeng Co., Ltd. The real-time PCR kit was purchased from Guangzhou Funeng Co., Ltd. PCR conditions were $95^{\circ} \mathrm{C}$ for 10 seconds, $60^{\circ} \mathrm{C}$ for 20 seconds, $72^{\circ} \mathrm{C}$ for 10 seconds. Each sample was analyzed in triplicate. Relative quantification of mRNA was performed using the comparative threshold cycles (CT) method. This value was used to plot the gene expression employing the formula $2^{-\Delta \Delta C T}$.

\section{Detection of protein expression by western blotting}

Expression of TMED2, IGF1R, IGF2, PI3K, AKT and GAPDH protein were analyzed by the Western blot method, as described [27-29]. The primary antibodies used included polyclonal rabbit anti-TMED2 (1:1000; HPA014060; Sigma, USA); polyclonal rabbit anti-IGF1R (1:1000; ab39398; Abcam Inc., Cambridge, MA, USA); polyclonal rabbit anti-IGF2 (1:1000; ab9574; Abcam Inc., Cambridge, MA, USA); anti-PI3K antibody rabbit polyclonal antibody $(1: 1000$, ab86714, Abcam Inc., Cambridge, MA, USA); anti-AKT2 antibody rabbit polyclonal antibody (1:1000, ab175354, Abcam Inc., Cambridge, MA, USA) and polyclonal rabbit antiGAPDH (1:1000; ab8245; Abcam Inc., Cambridge, MA, USA). The band density was analyzed using a gel imaging system and compared against an internal control.

\section{Cell proliferation assay}

Cell proliferation was determined using the EdU assay was performed using the Cell-Light TM EdU imaging detecting kit according to the instructions in the kit (Ruibo Biotechnology, Guangzhou, China). EdU is a thymidine analog that can be used to label cells undergoing DNA replication [30-32]. Cell proliferation was assessed by the CCK-8 assay (Beyotime, Shanghai, China, C0037) following to the manufacture's instruction. Cells were seeded into 96-well plates and cultured for an additional $24 \mathrm{~h}$. After treatment with OT, $10 \mu \mathrm{l}$ of the kit reagent was added and then incubated for another 2 h. O.D. value was read at $450 \mathrm{~nm}$ to obtain the final results [33-35].

\section{Wound healing assay and matrigel invasion assays}

Migration of SKOV3 and A2780 cells was analyzed using the wound-healing assay in vitro. Cells were seeded into 6-well plates and cultivated until $90 \%$ growth confluence. Wounds were afflicted by scraping the monolayer cells with a sterile pipette tip. At 0,24, 48 and 72 hours after the wounding, cells were observed under low power in an Olympus light microscope. The distance between two wounds were measured at each time point, and expressed as the average percent of wound closure as compared to that at zero time. Invasion of SKOV3 and A2780 cells was evaluated by Matrigel invasion assays. For Transwell invasion assays, the upper side of an $8 \mu \mathrm{m}$ pore, $6.5-\mathrm{mm}$ polycarbonate transwell filter (Corning, New York, NY) chamber was uniformly coated with Matrigel basement membrane matrix (BD Biosciences, Bedford, MA) for $2 \mathrm{~h}$ at $37^{\circ} \mathrm{C}$ before cells were added. A total of $5 \times 104$ cells were seeded into the top chamber of a trans-well filter (in triplicate) and incubated for 48 hours. Invasive cells on the lower side of the filter, were fixed in $4 \%$ paraformaldehyde, stained in $0.5 \%$ crystal violet (Beyotime), and counted using a microscope. A total of five fields were counted for each transwell filter. Each field was counted and photographed at $200 \times$ magnification.

\section{In vivo tumor xenograft study}

All procedures for animal experiments were approved by the Committee on the Use and Care on Animals (Southern Medical University, Guanzhou, China), and performed in accordance with the institution guidelines. Ovarian cancer SKOV3 cells were infected with indicated lentiviral vectors and injected $\left(1 \times 10^{6}\right.$ cells per mouse in $200 \mathrm{ul}$ ) subcutaneously into the left armpit of 6-week-old BALB/c nude mice (LV3-NC infected group, $\mathrm{n}=5$; LV3-1 infected group, $\mathrm{n}=5$ ). Twenty eight days later, animals were sacrificed to confirm the presence of tumors, and weigh the established tumors.

\section{Statistical analysis}

All statistical analyses were performed using SPSS software, version 17.0 (Chicago, IL). Each experiment was performed in triplicate. Statistical analysis was performed by Student's t-test or analysis of variance (ANOVA). Data were presented as Mean \pm standard deviation. Statistical significance was defined as a p-value less than 0.05 . 


\section{Abbreviations}

TMED, transmembrane emp24 domain; IGF2, insulin-like growth factor-2; IGF1R, type 1 insulin-like growth factor receptor; PI3K, phosphatidylinositide 3-kinase; miR-30a, microRNA 30a; LV3-NC, LV3shTMED2-negative control; LV3-1, LV3-shTMED2-1; LV3-2, LV3-shTMED2-2.

\section{ACKNOWLEDGMENTS AND FUNDING}

We thank members of our laboratory for technical help and stimulating discussions. This work was supported by grants from Science and Technology Program Project of Guangdong Province(2016A020215115); Special Funding for Production-Study-Research Cooperative Innovation Program of Huadu District, Guangzhou(HD15CXY006); Major Project for Health Care of Tianhe District, Guangzhou (201604KW010).

\section{CONFLICTS OF INTEREST}

There are no potential conflicts of interest to declare.

\section{REFERENCES}

1. Bonnon C, Wendeler MW, Paccaud JP, Hauri HP. Selective export of human GPI-anchored proteins from the endoplasmic reticulum. J Cell Sci. 2010; 123:1705-1715.

2. Castillon GA, Aguilera-Romero A, Manzano-Lopez J, Epstein S, Kajiwara K, Funato K, Watanabe R, Riezman H, Muñiz M. The yeast p24 complex regulates GPI-anchored protein transport and quality control by monitoring anchor remodeling. Mol Biol Cell. 2011; 22:2924-2936.

3. Jerome-Majewska LA, Achkar T, Luo L, Lupu F, Lacy E. The trafficking protein Tmed2/p24beta(1) is required for morphogenesis of the mouse embryo and placenta. Dev Biol. 2010; 341:154-166.

4. Xiong X, Lu Y, Zhang L, Wang B, Zhao Y, Wang XJ, Huo X, Shen Y, Liang Z, Chen M. Discovery of novel cell proliferation-enhancing gene by random siRNA library based combinatorial screening. Comb Chem High Throughput Screen. 2010; 13:798-806.

5. Kim JG, Kang MJ, Yoon YK, Kim HP, Park J, Song SH, Han SW, Park JW, Kang GH, Kang KW, Oh DY, Im SA, Bang YJ, et al. Heterodimerization of glycosylated insulinlike growth factor-1 receptors and insulin receptors in cancer cells sensitive to anti-IGF1R antibody. PLoS One. 2012; 7:e33322.

6. Moran T, Felip E, Keedy V, Borghaei H, Shepherd FA, Insa A, Brown H, Fitzgerald T, Sathyanarayanan S, Reilly JF, Mauro D, Hsu K, Yan L, Johnson DH. Activity of dalotuzumab, a selective anti-IGF1R antibody, in combination with erlotinib in unselected patients with non-small-cell lung cancer: a phase I/II randomized trial. Exp Hematol Oncol. 2014; 3:26.

7. Mu Q, Wang L, Yu F, Gao H, Lei T, Li P, Liu P, Zheng X, Hu X, Chen Y, Jiang Z, Sayari AJ, Shen J, Huang H. Imp2 regulates GBM progression by activating IGF2/PI3K/Akt pathway. Cancer Biol. Ther. 2015; 16:623-633.

8. Hu J, Meng Y, Yu T, Hu L, Mao M. Ubiquitin E3 ligase MARCH7 promotes ovarian tumor growth. Oncotarget. 2015; 6:12174-12187. https://doi.org/10.18632/ oncotarget.3650.

9. Tang Z, Li C, Kang B, Gao G, Li C, Zhang Z. GEPIA: a web server for cancer and normal gene expression profiling and interactive analyses. Nucleic Acids Res. 2017.

10. Huang GS, Brouwer-Visser J, Ramirez MJ, Kim $\mathrm{CH}$, Hebert TM, Lin J, Arias-Pulido H, Qualls CR, Prossnitz ER, Goldberg GL, Smith HO, Horwitz SB. Insulin-like growth factor 2 expression modulates Taxol resistance and is a candidate biomarker for reduced disease-free survival in ovarian cancer. Clin Cancer Res. 2010; 16:2999-3010.

11. Brana I, Berger R, Golan T, Haluska P, Edenfield J, Fiorica J, Stephenson J, Martin LP, Westin S, Hanjani P, Jones MB, Almhanna K, Wenham RM, et al. A parallel-arm phase I trial of the humanised anti-IGF-1R antibody dalotuzumab in combination with the AKT inhibitor MK-2206, the mTOR inhibitor ridaforolimus, or the NOTCH inhibitor MK-0752, in patients with advanced solid tumours. Br J Cancer. 2014; 111:1932-1944.

12. Cheng DL, Xiang YY, Ji LJ, Lu XJ. Competing endogenous RNA interplay in cancer: mechanism, methodology, and perspectives. Tumour Biol. 2015; 36:479-488.

13. Chiu HS, Martínez MR, Bansal M, Subramanian A, Golub TR, Yang X, Sumazin P, Califano A. High-throughput validation of ceRNA regulatory networks. BMC Genomics. 2017; 18:418.

14. Zhang R, Guo Y, Ma Z, Ma G, Xue Q, Li F, Liu L. Long non-coding RNA PTENP1 functions as a ceRNA to modulate PTEN level by decoying miR-106b and miR-93 in gastric cancer. Oncotarget. 2017; 8:26079-26089. https:// doi.org/10.18632/oncotarget.15317.

15. Martirosyan A, De Martino A, Pagnani A, Marinari E. ceRNA crosstalk stabilizes protein expression and affects the correlation pattern of interacting proteins. Sci Rep. 2017; 7:43673.

16. Li JH, Liu S, Zhou H, Qu LH, Yang JH. starBase v2.0: decoding miRNA-ceRNA, miRNA-ncRNA and proteinRNA interaction networks from large-scale CLIP-Seq data. Nucleic Acids Res. 2014; 42:D92-97.

17. Fu X, Li Y, Alvero A, Li J, Wu Q, Xiao Q, Peng Y, Hu Y, Li X, Yan W, Guo K, Zhou W, Wang Y, et al. MicroRNA222-3p/GNAI2/AKT axis inhibits epithelial ovarian cancer cell growth and associates with good overall survival. Oncotarget. 2016; 7:80633-80654. https://doi.org/10.18632/ oncotarget. 13017 . 
18. Linnerth-Petrik NM, Santry LA, Moorehead R, Jücker M, Wootton SK, Petrik J. Akt isoform specific effects in ovarian cancer progression. Oncotarget. 2016; 7:7482074833. https://doi.org/10.18632/oncotarget.11204.

19. Roberts CM, Tran MA, Pitruzzello MC, Wen W, Loeza J, Dellinger TH, Mor G, Glackin CA. TWIST1 drives cisplatin resistance and cell survival in an ovarian cancer model, via upregulation of GAS6, L1CAM, and Akt signalling. Sci Rep. 2016; 6:37652.

20. Dai Y, Jin S, Li X, Wang D. The involvement of Bcl-2 family proteins in AKT-regulated cell survival in cisplatin resistant epithelial ovarian cancer. Oncotarget. 2017; 8:1354-1368. https://doi.org/10.18632/oncotarget.13817.

21. Wei W, Yang Y, Cai J, Cui K, Li RX, Wang H, Shang $\mathrm{X}$, Wei D. MiR-30a-5p suppresses tumor metastasis of human colorectal cancer by targeting ITGB3. Cell Physiol Biochem. 2016; 39:1165-1176.

22. Wang LL, Zhang XH, Zhang X, Chu JK. MiR-30a increases cisplatin sensitivity of gastric cancer cells through suppressing epithelial-to-mesenchymal transition (EMT). Eur Rev Med Pharmacol Sci. 2016; 20:1733-1739.

23. Zhu J, Zeng Y, Li W, Qin H, Lei Z, Shen D, Gu D, Huang JA, Liu Z. CD73/NT5E is a target of miR-30a-5p and plays an important role in the pathogenesis of non-small cell lung cancer. Mol Cancer. 2017; 16:34.

24. Han X, Zhen S, Ye Z, Lu J, Wang L, Li P, Li J, Zheng X, Li H, Chen W, Li X, Zhao L. A feedback loop between miR-30a/c-5p and DNMT1 mediates cisplatin resistance in ovarian cancer cells. Cell Physiol Biochem. 2017; 41:973-986.

25. Meng F, Wang F, Wang L, Wong SC, Cho WC, Chan LW. MiR-30a-5p overexpression may overcome EGFR-inhibitor resistance through regulating PI3K/AKT signaling pathway in non-small cell lung cancer cell lines. Front Genet. 2016; $7: 197$.

26. Au CW, Siu MK, Liao X, Wong ES, Ngan HY, Tam KF, Chan DC, Chan QK, Cheung AN. Tyrosine kinase B receptor and BDNF expression in ovarian cancers - effect on cell migration, angiogenesis and clinical outcome. Cancer Lett. 2009; 281:151-161.

27. Hu J, Meng Y, Zhang Z, Yan Q, Jiang X, Lv Z, Hu L. MARCH5 RNA promotes autophagy, migration, and invasion of ovarian cancer cells. Autophagy. 2017; $13: 333-344$.

28. Zhao X, Fang Y, Yang Y, Qin Y, Wu P, Wang T, Lai H, Meng L, Wang D, Zheng Z, Lu X, Zhang H, Gao Q, et al. Elaiophylin, a novel autophagy inhibitor, exerts antitumor activity as a single agent in ovarian cancer cells. Autophagy. 2015; 11:1849-1863.

29. He J, Yu JJ, Xu Q, Wang L, Zheng JZ, Liu LZ, Jiang BH. Downregulation of ATG14 by EGR1-MIR152 sensitizes ovarian cancer cells to cisplatin-induced apoptosis by inhibiting cyto-protective autophagy. Autophagy. 2015; 11:373-384.

30. Ding D, Zhang Y, Wen L, Fu J, Bai X, Fan Y, Lin Y, Dai H, Li Q, Zhang Y, An R. MiR-367 regulates cell proliferation and metastasis by targeting metastasis-associated protein 3 (MTA3) in clear-cell renal cell carcinoma. Oncotarget. 2017; 8:63084-63095. https://doi.org/10.18632/ oncotarget. 18647 .

31. Tang G, Weng Z, Song J, Chen Y. Reversal effect of Jagged1 signaling inhibition on CCl4-induced hepatic fibrosis in rats. Oncotarget. 2017; 8:60778-60788. https:// doi.org/10.18632/oncotarget.18484.

32. Pereira PD, Serra-Caetano A, Cabrita M, Bekman E, Braga J, Rino J, Santus R, Filipe PL, Sousa AE, Ferreira JA. Quantification of cell cycle kinetics by EdU (5-ethynyl2'-deoxyuridine)-coupled-fluorescence-intensity analysis. Oncotarget. 2017; 8:40514-40532. https://doi.org/10.18632/ oncotarget.17121.

33. Li B, Jin X, Meng H, Hu B, Zhang T, Yu J, Chen S, Guo X, Wang W, Jiang W, Wang J. Morin promotes prostate cancer cells chemosensitivity to paclitaxel through miR-155/ GATA3 axis. Oncotarget. 2017; 8:47849-47860. https://doi. org/10.18632/oncotarget.18133.

34. Zhu W, Qian J, Ma L, Ma P, Yang F, Shu Y. MiR-346 suppresses cell proliferation through SMYD3 dependent approach in hepatocellular carcinoma. Oncotarget. 2017; 8:65218-65229. https://doi.org/10.18632/oncotarget.18060.

35. Zhang B, Yang Y, Tang J, Tao Y, Jiang B, Chen Z, Feng H, Yang L, Zhu G. Establishment of mouse neuron and microglial cell co-cultured models and its action mechanism. Oncotarget. 2017; 8:43061-43067. https://doi. org/10.18632/oncotarget.17898. 